\title{
Distinct polysomnographic and ECG- spectrographic phenotypes embedded within obstructive sleep apnea
}

\author{
Robert Joseph Thomas $^{1 *}$, Chol Shin ${ }^{2}$, Matt Travis Bianchi ${ }^{3}$, Clete Kushida ${ }^{4}$ and Chang-Ho Yun ${ }^{5}$
}

\begin{abstract}
Background: The primary metric extracted from the polysomnogram in patients with sleep apnea is the apnea-hypopnea index (or respiratory disturbance index) and its derivatives. Other phenomena of possible importance such as periods of stable breathing, features suggestive of high respiratory control loop gain, and sleep fragmentation phenotypes are not commonly generated in clinical practice or research. A broader phenotype designation can provide insights into biological processes, and possibly clinical therapy outcome effects.
\end{abstract}

Methods: The dataset used for this study was the archived baseline diagnostic polysomnograms from the Apnea Positive Pressure Long-term Efficacy Study (APPLES). The electrocardiogram (ECG)-derived cardiopulmonary coupling sleep spectrogram was computed from the polysomnogram. Sleep fragmentation phenotypes used thresholds of sleep efficiency (SE) $\leq 70 \%$, non-rapid eye movement (NREM) sleep N1 $\geq 30 \%$, wake after sleep onset (WASO) $\geq 60$ min, and high frequency coupling (HFC) on the ECG-spectrogram $\leq 30 \%$. Sleep consolidation phenotypes used thresholds of SE $\geq 90 \%$, WASO $\leq 30 \mathrm{~min}, \mathrm{HFC} \geq 50 \%$ and N1 $\leq 10 \%$. Multiple and logistic regression analysis explored cross-sectional associations with covariates and across phenotype categories. NREM vs. REM dominant apnea categories were identified when the NREM divided by REM respiratory disturbance index (RDI) was $>1$.

Results: The data was binned first into mild, moderate, severe and extreme categories based on the respiratory disturbance index of $<10,10-30,30-60$, and greater than 60, per hour of sleep. Using these criteria, 70, 394, 320 and 188 for polysomnogram, and 54, 296, 209 and 112 subjects for ECG-spectrogram analysis groups. All phenotypes were seen at all severity levels. There was a higher correlation of NREM-RDI with the amount of ECG-spectrogram narrow band coupling, vs. REM-RDI, 0.41 vs 0.14 , respectively. NREM dominance was associated with male gender and higher mixed/central apnea indices. Absence of the ECG-spectrogram sleep consolidated phenotype was associated with an increased odds of being on antihypertensive medications, OR 2.65 [Cl: 1.64-4.26], $p=<0.001$.

Conclusions: Distinct phenotypes are readily seen at all severities of sleep apnea, and can be identified from conventional polysomnography. The ECG-spectrogram analysis provides further phenotypic differentiation.

Keywords: Sleep apnea, Phenotypes, NREM-dominant, Sleep fragmentation, ECG-spectrogram,

Cardiopulmonary coupling

\footnotetext{
* Correspondence: rthomas1@bidmc.harvard.edu

${ }^{1}$ Division of Pulmonary, Critical Care, and Sleep Medicine, Beth Israel

Deaconess Medical Center, Boston, MA, USA

Full list of author information is available at the end of the article
} 


\section{Background}

Traditional sleep apnea morphological categories include obstructive, central, and periodic breathing/Cheyne-Stokes respiration types. Conventionally, polysomnogram (PSG) recordings are scored using $30 \mathrm{~s}$ epochs, into wake, rapid eye movement (REM) and non-rapid eye movement (NREM) stages. Respiratory events may be dominant in NREM or REM sleep, and at times be equally severe in both states. These events can be short, as at high altitude (25 s or less), or long, as in congestive heart failure (often over $60 \mathrm{~s}$ ). NREM stages are further characterized into grades, N1 through N3. Alternate methods of characterizing sleep include cyclic alternating pattern (CAP) of NREM sleep (Parrino et al. 2014), and cardiopulmonary coupling (high, low and very low frequency coupling of autonomic and respiratory drives, modulated by cortical delta power) (Thomas et al. 2014). Periods of stable breathing are usually associated with $\mathrm{N} 3$, and always associated with non-CAP and high frequency coupling.

The apnea-hypopnea index is the result of distinct interacting biological processes, all of which can contribute to the severity of clinical sleep apnea individually and collectively. These are high loop gain, low arousal threshold, airway collapsibility, and reduced negative pressure reflex response (Owens et al. 2015; Wellman et al. 2013; Eckert et al. 2013). A computational method to derive loop gain from routine PSG data was recently proposed, based on the concept that ventilatory fluctuations from apneas/hypopneas cause opposing changes in ventilatory drive according to the loop gain (Terrill et al. 2015). It would be more useful in clinical practice if there were features on the conventional PSG or metrics computed from PSG signals which differentiated phenotypes that could guide therapy. Specifically, a high loop gain phenotype may benefit from supplemental oxygen (Wellman et al. 2008), acetazolamide (Edwards et al. 2012), or hypocapnia minimization strategies, while sedatives could be an option in those who have low arousal thresholds in NREM sleep (Smales et al. 2015).

The conventional scoring criteria for central hypopnea strongly skews the events index to obstruction-most importantly, flow-limitation is frequently seen in periodic breathing, even at high altitude (Weiss et al. 2009), a quintessential model of high loop gain sleep apnea. High loop gain apnea is NREM dominant, regardless of the admixed obstructive features (Xie et al. 2011). Quantifying NREM vs. REM dominance may thus provide a pathophysiological phenotype. The oscillatory profile of respiratory oscillations or downstream respiration-driven or associated oscillations such as heart rate variability, blood pressure or even the electroencephalogram can be quantified (Maestri et al. 2010). We present use of a ECG-derived cardiopulmonary coupling analysis to detect high loop gain apnea independent of conventional scoring.
Respiration is stable during conventional slow wave sleep. The state of the cortical sleep network seems important (Thomas 2002). Increased genioglossus tone and increases in $\mathrm{CO}_{2}$ occur during periods of stable breathing (Jordan et al. 2009), with overt hypoventilation and hypoxia if flow limitation is severe during stable breathing periods. Central sleep apnea, periodic breathing, and treatment-emergent/complex apnea are NREM sleep phenomena. Stable breathing periods have traditionally not been quantified-the focus has been on the various thresholds and associations to determine clinically significant apnea or hypopnea. We quantified stable breathing using the ECG-based cardiopulmonary coupling technique, as described below. Stable breathing periods in apnea patients will likely demonstrate increased upper airway resistance. Despite the strong link between stable breathing and stage N3, the relationship is not exclusive: specifically, most periods of stable breathing occur in stage $\mathrm{N} 2$, even in those patients with no scored N3 sleep.

It is a common clinical observation that some patients with sleep apnea have disproportionate sleep fragmentation, and some with severe apnea demonstrate relatively intact macro-architecture of sleep. We used a well characterized sleep apnea clinical trial dataset, the Apnea Positive Pressure Long-term Efficacy Study (APPLES) (Kushida et al. 2006), to determine if at every severity of sleep apnea, discernable sleep and sleep apnea phenotypes exist. We used conventional polysomnogram metrics complemented with an electrocardiogram (ECG)based analysis that can detect periods of stable breathing (Thomas et al. 2005) and pathological respiratory chemoreflex activation (Thomas et al. 2007a).

\section{Methods \\ Database}

The APPLES data was obtained in Alice ${ }^{\mathrm{Tm}}$ and European Data Format, the latter was used for ECG-spectrogram analysis. He study randomized just over 1000 subjects to continuous positive airway pressure (CPAP) or placebo CPAP. A total of 972 baseline diagnostic polysomnograms were obtained; a subset of the data was embargoed by the primary study for administrative reasons. The following subjective and objective measures of sleepiness, mood and cognition were available: Hamilton Depression Scale, Epworth Sleepiness Scale, Stanford Sleepiness Scale, Paced Auditory Serial Addition Test, Psychomotor Vigilance Test Median and Mean reaction times, Maintenance of Wakefulness Test, and Short Term Working Memory.

\section{Polysomnogram scoring}

Standard scoring was done based on pre-2007 criteria on the polysomnogram data, generating respiratory, arousal, and sleep stage indices. The respiratory disturbance index 
used in the APPLES is equivalent to the current hypopnea definition with a $3 \%$ oxygen desaturation and/or arousal (Berry, 2017). We combined stage III and IV as the equivalent of current stage N3 (slow wave sleep). In this paper, we use the current terminology and designations to keep with current terms used in publications, though stage $\mathrm{N} 1$ and $\mathrm{N} 2$ will show some differences, typically greater N1, if the data were re-scored. A breakdown of REM and NREM RDI was also available. The characteristics of the full study population have also been published (Kushida et al. 2006; Quan et al. 2011). NREM vs. REM dominance was computed as the ratio of NREM/REM RDI; a value $>1$ was considered NREM dominance.

\section{ECG-spectrogram analysis}

The cardiopulmonary coupling (CPC) analysis (Figs. 1 and 2) of the ECG signal was performed as previously described in detail (Thomas et al. 2005). Briefly, heart rate variability and ECG-derived respiration (EDR; amplitude variations in the QRS complex due to shifts in the cardiac electrical axis relative to the electrodes during respiration and changes in thoracic impedance as the lungs fill and empty) are extracted from a single channel of ECG. Time series of normal-to-normal sinus $(\mathrm{N}-\mathrm{N})$ intervals and the time series of the EDR associated with these NN intervals are then extracted from the original $\mathrm{R}-\mathrm{R}$ (QRS to QRS) interval time series. Outliers due to false or missed R-wave detections are removed using a sliding window average filter with a window of 41 data points and rejection of central points lying outside $20 \%$ of the window average. The resulting NN interval series and its associated EDR are then resampled using cubic splines at $2 \mathrm{~Hz}$. The cross-spectral power and coherence of these two signals are calculated over a 1024 sample $(8.5 \mathrm{~min})$ window using the Fast Fourier Transform applied to the 3 overlapping 512 sample sub-windows within the 1024 coherence window. The 1024 coherence window is then advanced by 256 samples $(2.1 \mathrm{~min})$ and the calculation repeated until the entire NN interval/EDR series is analyzed. For each 1024 window the product of the coherence and cross-spectral power is used to calculate the ratio of coherent cross power in the low frequency $(0.01-0.1 \mathrm{~Hz}$.) band to that in the high frequency $(0.1-0.4 \mathrm{~Hz}$.) band. The logarithm of the high to low frequency cardiopulmonary coupling ratio $(\log [\mathrm{HFC} / \mathrm{LFC}])$ is then computed to yield a continuously varying measure of cardiopulmonary coupling. The graph of the amplitude of cardiopulmonary coupling at relevant frequencies (ordinate) vs. time (abscissa) provides a sleep spectrogram. Since the period of central apnea

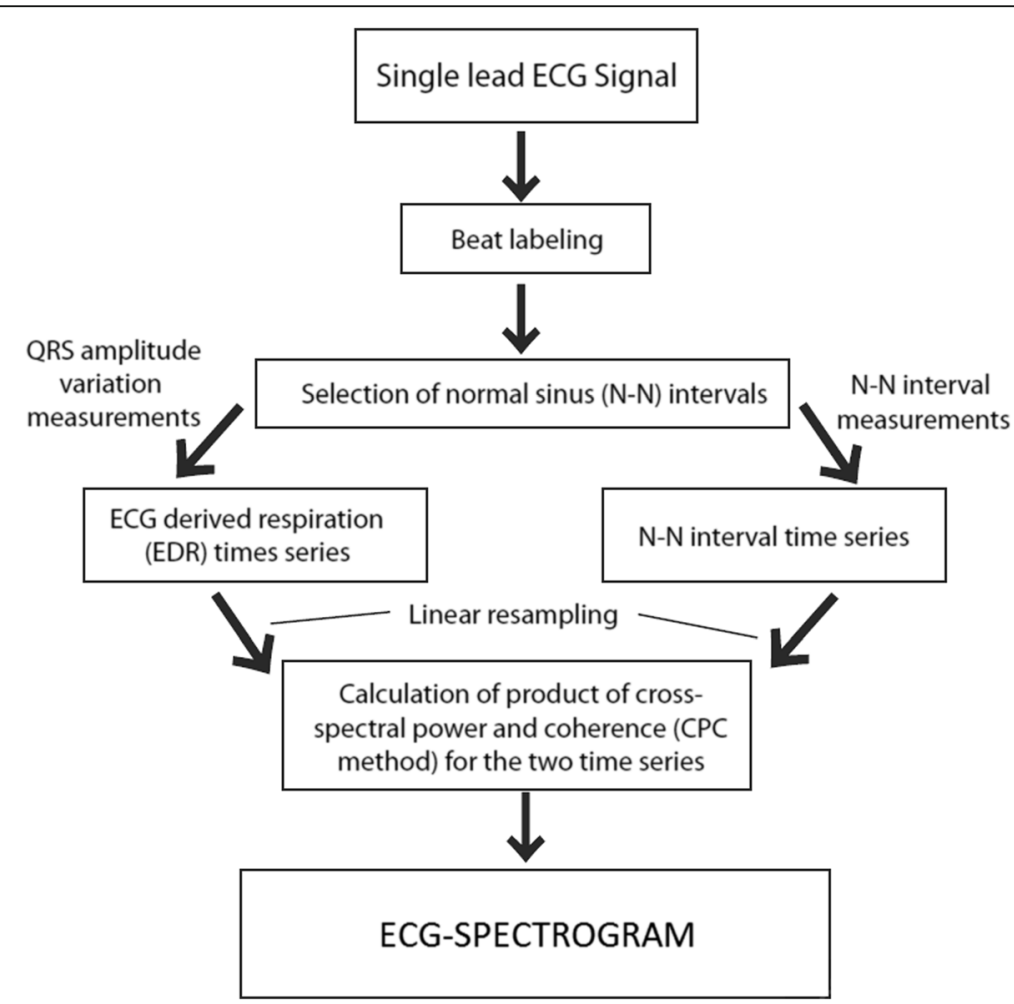

Fig. 1 Algorithm outline for the ECG-cardiopulmonary coupling analysis. The schema describes the analytic pathway for cardiopulmonary coupling analysis, using two distinct data streams embedded within the ECG: autonomic drive via heart rate variability and respiratory ECG-R amplitude modulation as a surrogate of respiration 


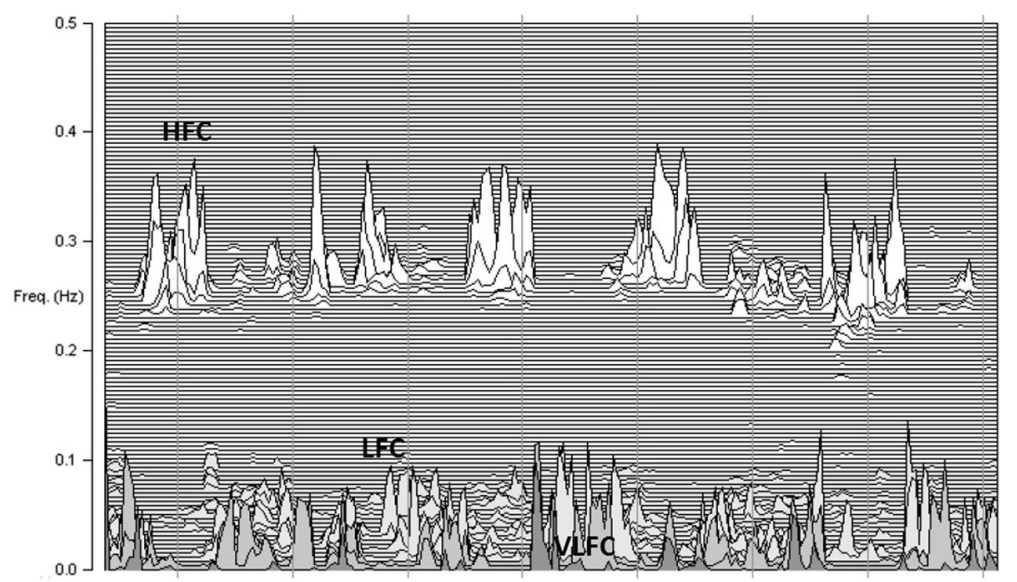

Fig. 2 Sample ECG-spectrogram. Note high, low and very low frequency coupling (HFC, LFC and VLFC respectively) and the clear separation in signal space of HFC from LFCNLFC. HFC is the ECG-spectrogram signal biomarker of stable breathing and stable sleep. VLFC reflects REM or wake

can be as slow as $120 \mathrm{~s}$ or longer, we used the frequency band between 0.006 and $0.1 \mathrm{~Hz}$ to define narrow spectral band e-LFC (putative central sleep apnea, periodic breathing, or complex sleep apnea). We required (1) a minimum power in this band of 0.3 normalized units and (2) that the coupling frequency of each pair of consecutive measurements remains within $0.0059 \mathrm{~Hz}$ of each other over 5 consecutive sampling windows (totaling 17 continuous min). Periods of e-LFC not meeting these criteria were defined as broad spectral band e-LFC (putative pure obstructive sleep apnea). The amounts of broad and narrow spectral band coupling in e-LFC bands were then expressed as the percentage of windows detected in relation to the total sleep period. Thus, the narrow spectral band e-LFC identified periods with oscillations that have a single dominant coupling frequency, suggesting central sleep apnea or periodic breathing (Thomas et al. 2007a). The broad spectral band e-LFC identified periods with oscillations that have variable coupling frequencies, suggesting an alternative mechanism, which we posited was dominance of anatomic upper airway obstructive processes. As it takes $17 \mathrm{~min}$ of continuous narrow-band cardiopulmonary coupling to reach the detection threshold, we estimated that this would be approximately equal to an averaged central apnea index of $5 / \mathrm{h}$ of sleep, assuming $6 \mathrm{~h}$ of sleep and a periodic breathing cycle length of approximately $35 \mathrm{~s}$. Finally, using the mean frequency and percentage of total sleep time in state, the LFC and e-LFC oscillation indices and mean cycle time were computed.

\section{Phenotype designation}

The data was binned first into mild, moderate, severe and extreme categories based on the respiratory disturbance index (respiratory events scored with a 3-s arousal or $4 \%$ oxygen desaturation) of $<10,10-30,30-60$, and greater than 60 , per hour of sleep. These severity groups were chosen to capture a range from mild to most severe. For example, $\geq 60$ could be considered "extreme", but would be subsumed otherwise if $\geq 30$ only was used as a "severe" cut off. Phenotype percentages were based on 972 and 617 subjects for polysomnographic and spectrographic phenotyping, respectively. Table 1 lists the criteria for the phenotypes. Figure 3 shows that individual phenotypes may or may not coexist. The criteria for a sleep fragmentation phenotype was based on clinical reasonableness, as no formal criteria exist. On a polysomnogram, a "fragmentation

Table 1 Phenotype Definitions

\begin{tabular}{|c|c|}
\hline Phenotype & Phenotype criteria \\
\hline Polysomnogram chemoreflex & Central apnea index $\geq 5 / h$ of sleep \\
\hline Spectrogram chemoreflex & $\begin{array}{l}\text { Presence of narrow band elevated } \\
\text { low frequency coupling }\end{array}$ \\
\hline $\begin{array}{l}\text { Polysomnogram } \\
\text { fragmentation-SE }\end{array}$ & $\begin{array}{l}\text { Sleep efficiency } \leq 70 \% \text { total sleep } \\
\text { time }\end{array}$ \\
\hline $\begin{array}{l}\text { Polysomnogram } \\
\text { fragmentation-N1 }\end{array}$ & NREM N1 $\geq 30 \%$ total sleep time \\
\hline $\begin{array}{l}\text { Polysomnogram } \\
\text { fragmentation -WASO }\end{array}$ & Wake after sleep onset $\geq 60 \mathrm{~min}$ \\
\hline Spectrogram fragmentation & $\begin{array}{l}\text { High frequency coupling } \leq 30 \% \\
\text { total sleep time }\end{array}$ \\
\hline $\begin{array}{l}\text { Polysomnogram } \\
\text { consolidation-SE }\end{array}$ & $\begin{array}{l}\text { Sleep efficiency } \geq 90 \% \text { total sleep } \\
\text { time }\end{array}$ \\
\hline $\begin{array}{l}\text { Polysomnogram } \\
\text { consolidation-N1 }\end{array}$ & NREM N1 $\leq 10 \%$ total sleep time \\
\hline $\begin{array}{l}\text { Polysomnogram } \\
\text { consolidation-WASO }\end{array}$ & Wake after sleep onset $\leq 30 \mathrm{~min}$ \\
\hline Spectrogram consolidation & $\begin{array}{l}\text { High frequency coupling } \geq 50 \% \\
\text { total sleep time }\end{array}$ \\
\hline
\end{tabular}

SE sleep efficiency; N1 NREM stage 1; WASO wake after sleep onset 

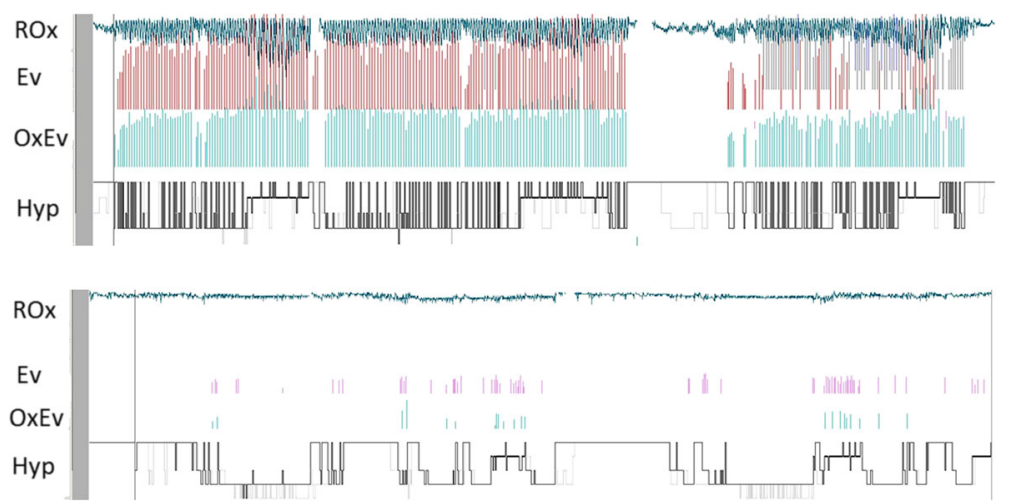

Fig. 3 Patterns of sleep fragmentation phenotype based on sleep efficiency. The upper hypnogram shows rapid sleep-wake transitions from severe sleep apnea, while the lower hypnogram shows nearly the same sleep efficiency but with consolidated periods of wake separated by consolidated periods of sleep. The \% N1 is markedly increased in the patient with rapid transitions (44.2\%). Thus, individual phenotypes can mix and match. ROx: raw oximetry. EV: respiratory events. OxEv: Oximetry desaturation events. Hyp: sleep stage hypnogram

phenotype" can be suggested by prolonged return to sleep following arousals/awakenings, low sleep efficiency $(<70 \%)$, high N1, and high wake after sleep onset (Thomas 2014). There is too little N3 to be a useful discriminatory metric. We choose a high frequency coupling $\%$ of $\geq 50$ and $\leq 30$ as thresholds for consolidated and fragmented phenotypes, guided by data from healthy individuals (Thomas et al. 2005) and analysis of the Sleep Heart Health Study (Thomas et al. 2014; Thomas et al. 2009).

\section{Statistical analysis}

Summary measures were mean/standard deviations for continuous measures, and proportions for categorical measures. T-tests were used to assess differences between NREM and REM dominance ratios. Logistic Regression with adjustment for age, gender, BMI, ethnicity and overall RDI assessed Odds Ratios for different phenotypic categories, with the following predictor categories: central apnea index, presence/absence of narrow band coupling, and the ECG-spectrogram sleep fragmentation category. The full multiple regression model assessing associations of individual phenotypes adjusted for age, gender, ethnicity, body mass index and sleep apnea severity (RDI), and total sleep time for PSG-based metrics. Pearson's Correlation estimated relationships between ECG spectrogram and polysomnogram indices. $\mathrm{Chi}^{2}$ test was used to assess significant differences of phenotypes across categories of apnea severity.

\section{Results}

\section{Demographics and polysomnography}

The demographic and polysomnographic characteristics of the subjects are described in Table 2, the 972 with polysomnogram and the 671 with ECG-spectrogram analysis. There were no significant differences. Loss of ECG-spectrogram analysis occurred from the following reasons: 1) Movement artifact or gaps, including bathroom breaks, $\geq 10 \mathrm{~min}$. Such dropouts made up the majority (86\%) of lost data. 2) Signal drop out, e.g., displaced electrode. There were no significant differences between included and excluded subjects, in terms of demographics and clinical conditions such as hypertension and diabetes. Central sleep apnea, defined as CAI $\geq 5 / h$ of sleep, was noted in 47/972 (5.1\%) and 30/671 (4.5\%), respectively.

\section{ECG-spectrogram characteristics}

Characteristics of the APPLES is described in Table 3. The mean cycle time calculated by the ECG spectrogram was just over $30 \mathrm{~s}$. There was a higher correlation of NREM-RDI with the amount of narrow band coupling, vs. REM-RDI, 0.41 vs 0.14 , respectively (Table 4).

\section{Polysomnographic and spectrographic phenotypes}

There were 70, 394, 320 and 188 subjects in the four categories of increasing severity (Tables 5 and 6). The corresponding sample size with the ECG-spectrogram was 54, 296, 209 and 112, respectively. The proportion of clinical phenotypes were different across severity in some but not all categories (Table 5). Specifically, the ECG-spectrogram categories did not show significant changes across severity, but the polysomnogram phenotype categories did. While no subject in the mild category had central sleep apnea, $8 \%$ did in the most severe category.

\section{NREM vs. REM dominance}

NREM dominance of sleep apnea was observed in $26.1 \%$ $(242 / 671)$ of the cohort. The characteristics of the NREM vs. REM dominance groups are in Table 7. Notable features associated with NREM dominance are: 1) 
Table 2 Polysomnographic and Demographic features

\begin{tabular}{|c|c|c|}
\hline $\begin{array}{l}\text { Demographics and } \\
\text { Polysomnogram Metrics }\end{array}$ & $\begin{array}{l}\text { Summary statistic } \\
\text { (972) Mean } \pm \text { SD }\end{array}$ & $\begin{array}{l}\text { Summary statistic } \\
\text { (671) Mean } \pm \text { SD }\end{array}$ \\
\hline Age & $50.8 \pm 12.9$ & $50.2 \pm 12.7$ years \\
\hline Gender & $65.3 \%$ male & $63.9 \%$ male \\
\hline Race & $70.2 \%$ white & $71.7 \%$ white \\
\hline Body Mass Index Kg/M² & $31.8 \pm 7.4$ & $31 \pm 7.1$ \\
\hline Total sleep time (TST) & $379.4 \pm 66.6$ & $385.1 \pm 60.3$ \\
\hline Sleep efficiency (\% TST) & $79 \pm 12.6$ & $80.1 \pm 11.6 \%$ \\
\hline $\begin{array}{l}\text { Wake After Sleep Onset } \\
\text { (minutes) }\end{array}$ & $83.4 \pm 51.8$ & $78.3 \pm 48.3$ \\
\hline S1 (\% TST) & $18.3 \pm 14.6$ & $17.3 \pm 13.1$ \\
\hline S2 (\% TST) & $60.3 \pm 13.4$ & $60.9 \pm 12.3$ \\
\hline S3 (\% TST) & $2.7 \pm 4.9$ & $2.7 \pm 4.9$ \\
\hline S4 (\% TST) & $0.7 \pm 2.4$ & $0.7 \pm 2.4$ \\
\hline REM (\% TST) & $17.9 \pm 7.1$ & $18.2 \pm 6.8$ \\
\hline Arousal Index/hour of sleep & $29 \pm 21.7$ & $27.6 \pm 20.8$ \\
\hline RDI/hour of sleep & $38.8 \pm 27.1$ & $36.4 \pm 26.1$ \\
\hline $\begin{array}{l}\text { Obstructive apnea index/ } \\
\text { hour of sleep }\end{array}$ & $16.9 \pm 21.9$ & $15.2 \pm 20.2$ \\
\hline $\begin{array}{l}\text { Central apnea index/hour } \\
\text { of sleep }\end{array}$ & $1.1 \pm 4.6$ & $\begin{array}{l}1.1 \pm 4.9 \\
\text { (Median 0.1, 25th/75th } \\
\text { percentile } 0.0 .1 \text { ) }\end{array}$ \\
\hline $\begin{array}{l}\text { Mixed apnea index/hour } \\
\text { of sleep }\end{array}$ & $1.5 \pm 5.6$ & $1.3 \pm 4.8$ \\
\hline $\begin{array}{l}\text { Hypopnea index/hour } \\
\text { of sleep }\end{array}$ & $19.3 \pm 13.2$ & $18.9 \pm 13$ \\
\hline RDI-NREM/hour of sleep & $37.4 \pm 28.9$ & $34.9 \pm 27.9$ \\
\hline RDI-REM/hour of sleep & $43.2 \pm 26.6$ & $42.1 \pm 26.5$ \\
\hline $\begin{array}{l}\text { Oxygen desaturation } \\
\text { index/hour of sleep }\end{array}$ & $25.5 \pm 25.6$ & $22.7 \pm 24$ \\
\hline Minimum saturation \% & $81 \pm 9.1$ & $81.7 \pm 8.4$ \\
\hline $\begin{array}{l}\text { Time less than } 85 \% \\
\text { saturation (minutes) }\end{array}$ & $2.9 \pm 8.2$ & $2.4 \pm 7.5$ \\
\hline PLM/hour of sleep & $6.4 \pm 15.4$ & $6 \pm 15$ \\
\hline
\end{tabular}

$R D /$ respiratory disturbance index; REM rapid eye movement; NREM non-rapid eye movement; PLM periodic limb movement

male gender; 2) greater degrees of sleep fragmentation; 3) more severe sleep apnea; 4) higher central and mixed apnea indices. The presence of narrow band coupling predicted NREM dominance, Odds Ratio 1.56 [CI: 1.1-2.29, p: 0.021], adjusted for age, gender, ethnicity, body mass index and overall RDI. Table 8 shows the predictors of NREM dominance, which included sleep fragmentation (positively) and ECG-sleep consolidation (negatively), adjusted for age, gender, BMI, ethnicity, and overall RDI.

\section{Predictors of phenotypes}

Correlation between the measures of sleep fragmentation or consolidation are to be expected. In our sample,
Table 3 ECG-spectrogram features in the APPLES

\begin{tabular}{lc}
\hline ECG-spectrogram metrics & $\begin{array}{c}\text { Mean } \pm \text { SD } \\
(n=671)\end{array}$ \\
\hline High frequency coupling \% TST & $38.9 \pm 22.3$ \\
High frequency coupling duration (minutes) & $151.4 \pm 92.7$ \\
Low frequency coupling \% TST & $43 \pm 20.2$ \\
Low frequency coupling duration (minutes) & $164.1 \pm 82$ \\
Elevated low frequency coupling \% TST & $20.8 \pm 17.9$ \\
Elevated low frequency coupling duration (minutes) & $79.4 \pm 69.3$ \\
Narrow band coupling \% TST & $3.4 \pm 8.1$ \\
Narrow band coupling duration (minutes) & $12.9 \pm 31.4$ \\
Very low frequency coupling \% TST & $16.1 \pm 7.3$ \\
Very low frequency coupling duration (minutes) & $61 \pm 28.2$ \\
CPC e-LFC index/hour of sleep & $20.6 \pm 19.3$ \\
CPC e-LFC cycle time (seconds) & $30.4 \pm 8.1$ \\
CPC LFC index/hour of sleep & $52.4 \pm 24.9$ \\
CPC LFC cycle time (seconds) & $31 \pm 8.4$ \\
\hline
\end{tabular}

TST total sleep time; CPC cardiopulmonary coupling; $L F C$ low frequency coupling; e-LFC elevated-low frequency coupling

the following were the $\mathrm{r}$ values, all $p:<0.001$ : WASO-N1 (0.33), WASO-sleep efficiency (-0.93), and N1-sleep efficiency $(-0.31)$. The categories of fragmentation or consolidation were related but also showed independence. After adjusting for age, gender, race, body mass index, total sleep time and sleep apnea severity, using logistic regression, the following were noted: 1) Sleep fragmentation: a) efficiency-N1: OR 1.89 [CI: 0.94-3.79], p: 0.072; b) efficiency-WASO: OR 4.19 [1.18-14.86], p: 0.027; c) N1WASO: OR 2.18 [1.24-3.83], p: 0.007. 2) Sleep consolidation: a) efficiency-N1: OR 2.6 [CI: 1.68-4.03], $p$ : < 0.001; b) efficiency-WASO: OR 50.19 [24.26-103.84], $p$ : < 0.001; c) N1-WASO: OR 1.96 [0.97-3.05], p: 0.07.

In a multiple regression analysis adjusted for age, gender, ethnicity, body mass index and sleep apnea severity (RDI), age was a consistent positive predictor of sleep efficiency, wake after sleep onset and N1 fragmentation categories. Coefficient \pm SE, p was $0.007 \pm 0.001 ; p$ : < $0.001 ; 0.015 \pm 0.001, p:<0.001$; and $0.004 \pm 0.001, p:<$ 0.001 , respectively. Male sex was predictive for N1 sleep fragmentation category; $0.06 \pm 0.03, \mathrm{p}: 0.020$. Central apnea category was predicted by age $(0.001 \pm 0.001$ per year, p: 0.024) and male sex (0.048 \pm 0.016 , p: 0.003$)$.

In a logistic regression analysis (Table 9), the central apnea category increased the odds of ECG-spectrogram fragmentation phenotype, and reduced that of the N1 PSG consolidation phenotype. The presence of narrow band coupling increased the odds of the $\mathrm{N} 1$ and the ECG-spectrogram fragmentation phenotypes, while reducing the odds of the ECG-spectrogram consolidation phenotype. Finally, the ECG-spectrogram fragmentation phenotype increased the odds of the N1 fragmentation 
Table 4 Correlation ( $r$ ) of ECG-spectrogram and PSG respiratory indices

\begin{tabular}{|c|c|c|c|c|c|}
\hline CPC metric & RDI & NREM-RDI & REM-RDI & CAl/hour of sleep & $\begin{array}{l}\text { Desaturation Index/hour } \\
\text { of sleep }\end{array}$ \\
\hline LFC index & $\begin{array}{l}0.50 \\
{[p: 0.021}\end{array}$ & $\begin{array}{l}0.52 \\
\text { [p: } 0.024]\end{array}$ & $\begin{array}{l}0.20 \\
\text { [p: } 0.043]\end{array}$ & $\begin{array}{l}0.21 \\
{[p: 0.041]}\end{array}$ & $\begin{array}{l}0.44 \\
\text { (p: } 0.001)\end{array}$ \\
\hline e-LFC index & $\begin{array}{l}0.56 \\
\text { [p: } 0.02]\end{array}$ & $\begin{array}{l}0.59 \\
{[p: 0.001]}\end{array}$ & $\begin{array}{l}0.22 \\
{[p: 0.041]}\end{array}$ & $\begin{array}{l}0.25 \\
{[p: 0.031]}\end{array}$ & $\begin{array}{l}0.50 \\
{[p: 0.001]}\end{array}$ \\
\hline$e-L F C_{N B}$ & $\begin{array}{l}0.39 \\
{[p: 0.021]}\end{array}$ & $\begin{array}{l}0.41 \\
{[p: 0.001]}\end{array}$ & $\begin{array}{l}0.14 \\
{[p: 0.071]}\end{array}$ & $\begin{array}{l}0.19 \\
\text { [p: } 0.043]\end{array}$ & $\begin{array}{l}0.36 \\
P:[0.013]\end{array}$ \\
\hline LFC cycle time & $\begin{array}{l}-0.01 \\
\text { [p: } 0.601]\end{array}$ & $\begin{array}{l}-0.01 \\
{[p: 0.311]}\end{array}$ & $\begin{array}{l}0.01 \\
{[p: 0.212]}\end{array}$ & $\begin{array}{l}-0.01 \\
{[p: 0.311]}\end{array}$ & $\begin{array}{l}-0.02 \\
{[p: 0.511]}\end{array}$ \\
\hline e-LFC cycle time & $\begin{array}{l}0.09 \\
\text { [p: } 0.413]\end{array}$ & $\begin{array}{l}0.09 \\
\text { [p: } 0.211]\end{array}$ & $\begin{array}{l}0.08 \\
\text { [p: } 0.412]\end{array}$ & $\begin{array}{l}0.02 \\
\text { [p: } 0.121]\end{array}$ & $\begin{array}{l}0.41 \\
\text { [p: } 0.013]\end{array}$ \\
\hline
\end{tabular}

LFC low frequency coupling; e-LFC elevated low frequency coupling; $R D /$ respiratory disturbance index; REM rapid eye movement sleep; NREM non-rapid eye movement sleep; CAl central apnea index; CPC cardiopulmonary coupling

phenotype and reduced the odds of the N1 and WASO consolidation phenotypes.

\section{Cycle time influences}

The cycle time of LFC $(30.4 \pm 8.1)$ and e-LFC $(31 \pm 8.4)$ was not significantly different across various sleep fragmented or consolidated phenotypes, or NREM vs. REM dominance (the latter $31 \pm 8.8$ vs. $31 \pm 8.2$, p: 0.99 ). Those with narrow-band coupling had a shorter cycle time than those without, $27.5 \pm 5.7$ vs. $32.8 \pm 8.8$ s, $p:<0.001$ for LFC, and $27.8 \pm 6.8$ vs. $32.6 \pm 8.7 \mathrm{~s}, p:<0.001$, for e-LFC.

\section{Phenotypes and clinical baseline covariates}

The total Epworth Sleepiness Scale was modified by NREM vs. REM dominance: $9.2 \pm 4.2$ vs. $10.2 \pm 4.3, t$ - test, p: 0.009. A multiple regression with adjustment for age, gender, body mass index, ethnicity, and total RDI remained significant: Beta Coefficient $-0.003 \pm$ SE 0.38, p: 0.009. Absence of the ECG-spectrogram sleep consolidated phenotype was associated with an increased odds of being on antihypertensive medications, OR 2.65 [CI: 1.64-4.26], $p:<0.001$, adjusted for age, gender, BMI, total sleep time, and slow wave sleep (pre 2007 stages S3 + S4). The difference in high frequency coupling in those with and without the ECG-spectrogram consolidated phenotype was substantial and clinically meaningful, $50.7 \pm 22.4$ vs. $37.3 \pm 21.8 \%$ total sleep time. Evening and morning systolic and diastolic blood pressures were, however, not significantly different. Other phenotypes at baseline including all cognitive measures did not show

Table 5 Polysomnographic and spectrographic metrics across apnea severity groups (mean \pm SD)

\begin{tabular}{|c|c|c|c|c|}
\hline Polysomnographic variable & Mild $(n=70)$ & Moderate $(n=394)$ & Severe $(n=320)$ & Extreme $(n=188)$ \\
\hline Total Sleep Time (minutes) & $386 \pm 68$ & $376 \pm 64$ & $388 \pm 63$ & $368 \pm 74$ \\
\hline Sleep efficiency \% TST & $80.9 \pm 13.3$ & $79 \pm 12.8$ & $80.1 \pm 11.1$ & $76.4 \pm 14.2$ \\
\hline Arousal index/hour of sleep & $14.2 \pm 10.4$ & $19.8 \pm 12.1$ & $29 \pm 15.3$ & $53.9 \pm 28.6$ \\
\hline S1 \% TST & $11.4 \pm 7.9$ & $14 \pm 8.4$ & $18.1 \pm 11.2$ & $30.6 \pm 22.5$ \\
\hline S2 \% TST & $63.6 \pm 8.4$ & $62.6 \pm 9.7$ & $60.6 \pm 11$ & $53.7 \pm 20.9$ \\
\hline $\mathrm{S} 3+\mathrm{S} 4 \% \mathrm{TST}$ & $5.5 \pm 7.2$ & $4 \pm 6.7$ & $3 \pm 5.4$ & $1.7 \pm 4.3$ \\
\hline REM \% TST & $19.4 \pm 6.6$ & $19.2 \pm 6.5$ & $18.2 \pm 6.8$ & $13.9 \pm 7.2$ \\
\hline $\mathrm{RDI} /$ hour of sleep & $5.5 \pm 2.7$ & $19.1 \pm 5.8$ & $44 \pm 8.7$ & $83.6 \pm 17.6$ \\
\hline CAl/hour of sleep & $0.8 \pm 0.2$ & $0.3 \pm 1$ & $1.2 \pm 3$ & $3.2 \pm 9.3$ \\
\hline Minimum $\mathrm{SaO}_{2}$ & $87.7 \pm 11.5$ & $84.8 \pm 5.1$ & $79.7 \pm 7.5$ & $72.7 \pm 10.6$ \\
\hline ECG-spectrographic variable & $n=54$ & $n=296$ & $n=209$ & $n=112$ \\
\hline HFC \% TST & $50.8 \pm 19.6$ & $44.5 \pm 21$ & $36.9 \pm 20.8$ & $22.1 \pm 19.7$ \\
\hline LFC \% TST & $29.4 \pm 13.8$ & $35.7 \pm 16.2$ & $45.6 \pm 17.8$ & $62.8 \pm 21$ \\
\hline e-LFC \% TST & $11.1 \pm 10.1$ & $15.3 \pm 11.5$ & $22.3 \pm 16.2$ & $37.3 \pm 25.2$ \\
\hline $\mathrm{e}-\mathrm{LFC} C_{N B} \% \mathrm{TST}$ & $0.02 \pm 0.07$ & $1.2 \pm 2.7$ & $3.8 \pm 8.5$ & $9.9 \pm 13.3$ \\
\hline VLFC \% TST & $17.6 \pm 7.1$ & $17.6 \pm 7.4$ & $15.2 \pm 6.9$ & $12.9 \pm 6.9$ \\
\hline
\end{tabular}

WASO wake after sleep onset; N1 stage 1 NREM sleep; CAI central apnea index; S2-S4 pre-2007 NREM sleep stages; HFC high frequency coupling; REM rapid eye movement; $L F C$ low frequency coupling; VLFC very low frequency coupling; $e-L C F$ elevated-low frequency coupling; $e-L F C_{N B}$ narrow band e-LFC 
Table 6 Phenotypes across sleep apnea severity categories

\begin{tabular}{|c|c|c|c|c|c|}
\hline Phenotype across apnea severity categories & Mild & Moderate & Severe & Extreme & $\begin{array}{l}\text { Chi }^{2}, p \text {, for phenotypes } \\
\text { across categories }\end{array}$ \\
\hline Chemoreflex phenotype_PSG (CAI $\geq 5$ /hour of sleep) & $0(0 \%)$ & $1(0.25 \%)$ & $13(4.1 \%)$ & $15(8 \%)$ & $\begin{array}{l}44.86 \\
<0.001\end{array}$ \\
\hline Chemoreflex phenotype—spectrogram (e-LFC $C_{N B}$ present) & $4(7.4 \%)$ & $70(23.7 \%)$ & $75(23.4 \%)$ & $67(35.6 \%)$ & $\begin{array}{l}4.83 \\
0.19\end{array}$ \\
\hline Sleep fragmentation phenotype-sleep efficiency $\leq 70 \%$ & $13(18.6 \%)$ & $82(20.8 \%)$ & $53(16.6 \%)$ & $48(25.5 \%)$ & $\begin{array}{l}6.16 \\
0.10\end{array}$ \\
\hline Sleep fragmentation phenotype-N1 $\geq 30 \%$ & $3(4.3 \%)$ & $24(6.1 \%)$ & $45(14.1 \%)$ & $76(40.4 \%)$ & $\begin{array}{l}124.78 \\
<0.001\end{array}$ \\
\hline Sleep fragmentation phenotype-WASO $\geq 60 \mathrm{~min}$ & $\begin{array}{l}38 \\
(54.3 \%)\end{array}$ & $\begin{array}{l}240 \\
(60.9 \%)\end{array}$ & $\begin{array}{l}196 \\
(61.3 \%)\end{array}$ & $\begin{array}{l}136 \\
(72.3 \%)\end{array}$ & $\begin{array}{l}10.42 \\
0.02\end{array}$ \\
\hline Sleep fragmentation phenotype—spectrogram (HFC $\leq 30 \%)$ & $9(16.7 \%)$ & $75(25.3 \%)$ & $80(38.3 \%)$ & $79(70.5 \%)$ & \\
\hline Sleep consolidation phenotype_-sleep efficiency $\geq 90 \%$ & $22(31.4 \%)$ & $76(19.3 \%)$ & $55(17.2 \%)$ & $27(14.4 \%)$ & $\begin{array}{l}9.89 \\
0.02\end{array}$ \\
\hline Sleep consolidation phenotype-N1 $\leq 10 \%$ & $42(60 \%)$ & $157(39.9 \%)$ & $85(26.6 \%)$ & $33(17.6 \%)$ & $\begin{array}{l}58.01 \\
<0.001\end{array}$ \\
\hline Sleep consolidation phenotype-WASO $\leq 30$ min & $\begin{array}{l}15 \\
21.4 \%)\end{array}$ & $\begin{array}{l}57 \\
(14.5 \%)\end{array}$ & $\begin{array}{l}34 \\
(10.6 \%)\end{array}$ & $\begin{array}{l}15 \\
(8 \%)\end{array}$ & $\begin{array}{l}11.08 \\
0.01\end{array}$ \\
\hline Sleep consolidation phenotype—spectrogram (HFC $\geq 50 \%$ ) & $28(51.9 \%)$ & $126(42.6 \%)$ & $61(29.2 \%)$ & $15(12.3 \%)$ & $\begin{array}{l}2.46 \\
0.48\end{array}$ \\
\hline
\end{tabular}

WASO wake after sleep onset; $N 1$ stage 1 NREM sleep; $C A I$ central apnea index; HFC high frequency coupling; $L F C$ low frequency coupling; $e-L F C_{N B}$ narrow band e-LFC

Table 7 NREM vs. REM dominance: clinical and polysomnographic features

\begin{tabular}{|c|c|c|c|}
\hline Demographics and Polysomnogram Metrics & NREM dominant (242) & REM dominant (429) & $\mathrm{p}$ \\
\hline Age (years) & $51.2 \pm 13.9$ & $49.6 \pm 11.9$ & 0.11 \\
\hline Gender (male \%) & 80.2 & 55.7 & $<0.001\left(\mathrm{Chi}^{2}\right)$ \\
\hline Race (white, \%) & 74.8 & 69.9 & 0.10 \\
\hline Body Mass Index Kg/M2 & $30.6 \pm 6.7$ & $31.3 \pm 7.3$ & 0.25 \\
\hline Total sleep time (TST) & $282.8 \pm 64$ & $386.4 \pm 58.1$ & 0.46 \\
\hline Sleep efficiency \% TST & $79.2 \pm 12.2$ & $80.7 \pm 11.1$ & 0.10 \\
\hline S1 \% TST & $21.7 \pm 16.8$ & $14.9 \pm 10.1$ & $<0.001$ \\
\hline S2 \% TST & $58.3 \pm 15.5$ & $62.3 \pm 9.8$ & $<0.001$ \\
\hline $\mathrm{S} 3+\mathrm{S} 4 \% \mathrm{TST}$ & $2.1 \pm 3.8$ & $3.1 \pm 5.3$ & 0.005 \\
\hline REM & $17.1 \pm 6.7$ & $18.9 \pm 6.7$ & 0.002 \\
\hline Arousal Index/hour of sleep & $36.5 \pm 26.1$ & $22.6 \pm 15.1$ & $<0.001$ \\
\hline RDI/hour of sleep & $49.9 \pm 29.9$ & $28.8 \pm 20.1$ & $<0.001$ \\
\hline Obstructive apnea index/hour of sleep & $25 \pm 15.4$ & $9.6 \pm 13.7$ & $<0.001$ \\
\hline Central apnea index/hour of sleep & $2 \pm 7.2$ & $0.5 \pm 2.1$ & $<0.001$ \\
\hline Mixed apnea index/hour of sleep & $3 \pm 7.4$ & $0.4 \pm 1.5$ & $<0.001$ \\
\hline Hypopnea index/hour of sleep & $19.9 \pm 14.5$ & $18.3 \pm 11.9$ & 0.11 \\
\hline RDI-NREM/hour of sleep & $52.9 \pm 30.5$ & $24.7 \pm 20.3$ & $<0.001$ \\
\hline RDI-REM/hour of sleep & $34.5 \pm 25.3$ & $46.5 \pm 25.3$ & $<0.001$ \\
\hline Oxygen desaturation index/hour of sleep & $34 \pm 29.6$ & $16.4 \pm 17.2$ & $<0.001$ \\
\hline Minimum saturation & $79.5 \pm 10.3$ & $82.9 \pm 6.8$ & $<0.001$ \\
\hline Time less than $85 \%$ saturation (minutes) & $4.8 \pm 11.5$ & $1 \pm 2.6$ & $<0.001$ \\
\hline PLM/hour of sleep & $5.8 \pm 12.5$ & $6.2 \pm 16.2$ & 0.77 \\
\hline
\end{tabular}

All values mean and standard deviation, unless other specified

S1-S4 Pre-2007 NREM sleep stages; RDI respiratory disturbance index; REM rapid eye movement NREM: non-rapid eye movement; PLM periodic limb movement 
Table 8 Predictors of NREM dominance

\begin{tabular}{|c|c|}
\hline Phenotype & NREM dominance $(\mathrm{OR}), \mathrm{p}$ \\
\hline PSG SF-SE & 1.16 [Cl: $0.72-1.86], 0.527$ \\
\hline PSG SF-N1 & 1.72 [Cl: $1.01-2.93], 0.046^{*}$ \\
\hline PSG SF-WASO & 0.90 [Cl: $0.61-1.34], 0.614$ \\
\hline ECG SF & 1.53 [Cl: $1.04-2.25], 0.029^{*}$ \\
\hline PSG SC-SE & 0.92 [Cl: $0.58-1.50], 0.758$ \\
\hline PSG SC-N1 & 0.91 [Cl: $0.61-1.35], 0.643$ \\
\hline PSG SC-WASO & 0.81 [Cl: $0.47-1.43], 0.483$ \\
\hline ECG SC & 0.44 [Cl: $0.29-0.67],<0.001^{*}$ \\
\hline Central sleep apnea + & 1.96 [Cl: $0.78-4.91], 0.152$ \\
\hline e-LFC $C_{N B}+$ & 1.56 [Cl: $1.07-2.29], 0.022$ \\
\hline
\end{tabular}

Adjusted for age, gender, body mass index, ethnicity, respiratory

disturbance index

Cl confidence intervals; NREM non-rapid eye movement sleep PSG: polysomnographic; SE sleep efficiency; N1 NREM stage 1; WASO wake after sleep onset; ECG electrocardiogram-based analysis; SC sleep consolidation; SF sleep fragmentation; $e-L F C_{N B}$ elevated-low frequency coupling narrow band * Statistically significant

differences in clinical covariates. The evening systolic blood pressure was higher in the NREM-dominant group, $125.3 \pm 14.7$ vs. $122.6 \pm 14.7 \mathrm{~mm} \mathrm{Hg}$, but was no longer significant after adjustment for age.

\section{CPAP compliance}

Use of CPAP during the last month of the 6-month trial was $4.2 \pm 2.2 \mathrm{~h}$, and $3.9 \pm 2.1 \mathrm{~h}$ across the entire duration. The percentage of use of CPAP for 4 or more hours, average of all subjects, was $55 \pm 20 \%$.

Mean compliance across the 6 months was lower in those with the sleep fragmentation-N1 group, $3.6 \pm 2$ vs. $4 \pm 2.1 \mathrm{~h}$. Other categories had no impact.

\section{Discussion}

The results of our analysis show that discernable phenotypes are present within what is otherwise considered generic obstructive sleep apnea. Fragmented and consolidated phenotypes are see at milder and more severe extremes of obstructive sleep apnea, using both conventional and computed analysis of polysomnogram signals. Presumptive high loop gain phenotypes, central sleep apnea on polysomnography and narrow-band coupling on ECG-spectrogram, are associated with greater degrees of sleep fragmentation. Stage dominance, NREM vs. REM shows clear differences. Cycle time metrics provide further insight into pathological interactions that result in a final common output, that of an apneahypopnea index. Several aspects of extractable phenotypes provide novel insights into sleep apnea.

\section{Periods of stable breathing during NREM sleep}

Some clues to the nature of this phenomenon can be gained from the concept of NREM sleep bimodality. The first clue came from the description of CAP and non-CAP from Italian researchers in the mid 1980's (Terzano et al. 1985). CAP and non-CAP periods occur across NREM sleep. CAP occurs in N1 and parts of N2; non-CAP occurs in parts of N2 and most of N3 (Parrino et al. 2014). Subsequently, the autonomic and respiratory associations of CAP/non-CAP were described (Kara et al. 2003). Finally, the description of the cardiopulmonary coupling technique showed that NREM sleep has bimodal characteristics in health and disease. High frequency coupling is associated with high delta power, non-CAP EEG, stable breathing, strong sinus arrhythmia, and blood pressure dipping (Thomas et al. 2014). Low frequency coupling is associated with unstable breathing, cyclic variation in heart rate, CAP EEG, and blood pressure non-dipping. Thus, stable breathing periods reflect natural integrated network states of the brain. Benzodiazepines and related drugs increase non-CAP (Parrino et al. 1997; Terzano et al. 1995), and may be expected to increase stable breathing periods. Zolpidem increases blood pressure dipping (Huang et al. 2012), and could do so through the induction of stable NREM periods.

Table 9 Predictors of Primary Phenotypes

\begin{tabular}{|c|c|c|c|}
\hline Phenotype & $\mathrm{CAl}(\mathrm{OR}), \mathrm{p}$ & $e-L F C_{N B}(O R), p$ & ECG SF (OR), p \\
\hline PSG SF-SE & 1.03 [Cl: 0.41-2.62], 0.939 & 0.88 [Cl: 0.56-1.37], 0.566 & 1.37 [Cl: $0.90-1.12], 0.144$ \\
\hline PSG SF-N1 & 1.70 [Cl:0.71-4.09], 0.228 & $2.97[\mathrm{Cl}: 1.86-4.74],<0.001^{*}$ & 1.95 [Cl:1.22-3.13], 0.005* \\
\hline PSG SF-WASO & 1.07 [Cl:0.46-2.48], 0.882 & 0.99 [Cl:0.69-1.43], 0.975 & 1.34 [Cl:0.93-1.93], 0.111 \\
\hline ECG SF & 3.69 [Cl:1.58-8.64], 0.003* & $2.06[\mathrm{Cl}: 1.47-2.93],<0.001^{*}$ & \\
\hline PSG SC-SE & 0.51 [Cl:0.14-1.78], 0.288 & 0.89 [Cl:0.57-1.38], 0.593 & 0.72 [Cl:0.46-1.13], 0.155 \\
\hline PSG SC-N1 & 0.26 [Cl:0.08-0.87], 0.029* & 0.64 [Cl:0.44-0.92], 0.018 & 0.61 [Cl:0.42-0.88], 0.009* \\
\hline PSG SC—WASO & 0.25 [Cl:0.03-1.91], 0.182 & 1.01 [Cl:0.61-1.69], 0.955 & 0.57 [Cl:0.33-0.97], 0.040* \\
\hline ECG SC & 0.31 [Cl:0.09-1.04], 0.057 & 0.28 [Cl:0.18-0.43], <0.001* & \\
\hline
\end{tabular}

Cl confidence intervals; PSG polysomnogram; ECG electrocardiogram; N1 NREM stage 1; SF sleep fragmentation; WASO wake after sleep onset; SC sleep consolidation; SE sleep efficiency; $C A l$ central apnea index; $e-L F C_{N B}$ elevated-low frequency coupling, narrow band

* Statistically significant 
The proportion of stable breathing periods will impact the computed apnea-hypopnea index, as these periods do not contribute to the metric. Varying proportions of stable breathing on a night to night basis can contribute to night to night variability of the apnea-hypopnea index. The fact that even at the most severe end of the spectrum there are patients with consolidated sleep by any measure suggests that this is an individual trait.

\section{NREM vs. REM dominance}

In general, periodic breathing and hypocapnic central apnea does not occur in REM sleep (exception, a patient with congestive heart failure who demonstrates periodic breathing during wake state). NREM dominance is well described in idiopathic central sleep apnea (Quadri et al. 2009), periodic breathing associated with heart failure or stroke (Hanly et al. 1989), opiate-induced sleep apnea (Walker et al. 2007), and high altitude periodic breathing (Thomas et al. 2007b). NREM dominance is also a feature of complex apnea/treatment-emergent central sleep apnea, regardless of the exact definition used (Rao \& Thomas 2013). In the APPLES data, NREM dominance was associated with greater severity of disease, male sex, and increased central/mixed apneas, even if less than the conventional threshold for central sleep apnea was used (which requires $\geq 50 \%$ of all events to be central). It is possible that NREM dominant obstructive sleep apnea reflects high loop gain (Rao \& Thomas 2013; Stanchina et al. 2015), and is thus a recognizable phenotype from standard polysomnograms even without further computational analysis.

\section{Accurate estimation of central sleep apnea from clinical polysomnograms}

In the APPLES data, the amount of central apnea/presumed high loop gain features estimated by conventional features vs. ECG-spectrogram diverged markedly. The American Academy of Sleep Medicine (AASM) criteria defines a central apnea as an oronasal flow drop by $>90 \%$ of baseline, lasting $10 \mathrm{~s}$, in the absence of inspiratory effort. (Iber \& American Academy of Sleep Medicine 2007) A central hypopnea requires proportional and concordant flow and effort reduction and absence of snoring (except possibly at recovery) and flow-limitation. However, events both at sea level (often) and high altitude (always) in patients with positive pressure induced or amplified respiratory instability have short cycles that are less than $30 \mathrm{~s}$. If $40 \mathrm{~s}$ is a requirement, then these short-cycle hypopnea events will be falsely characterized as obstructive. The International Classification of Sleep Disorders (ICSD)-3 specifies that these should make up $\geq 50 \%$ of all scored events, so substantial central events can still carry an obstructive summary label.
The scoring guidelines state that flow limitation excludes a "central hypopnea" in the scoring manual yet several lines of evidence argue strongly against this: a) at high-altitude, a pure chemoreflex form of sleep apnea, flow-limitation occurs frequently; b) studies using esophageal manometry and endoscopy show that pharyngeal airway narrowing and occlusion occur during central apneas in healthy individuals as well as in patients with heart failure. c) the airway can close during polysomnographic central apnea; (Badr 1996; Badr et al. 1995) d) central hypopneas demonstrate flow-limitation (Badr et al. 1995; Sankri-Tarbichi et al. 2009; Guilleminault et al. 1997; Dowdell et al. 1990). Despite the known presence of flow limitation and airway narrowing during both central and obstructive events (Dempsey et al. 2014), hypopnea scoring is biased towards obstructive disease (Rao \& Thomas 2013; Eckert et al. 2007; Javaheri \& Dempsey 2013).

The APPLES scoring did not include central hypopneas and thus likely underestimated high loop gain features. The ECG-spectrogram analysis showed that central/periodic breathing-type oscillations were present in nearly one third of the APPLES cohort, and that this signal biomarker was more closely associated with NREM than REM RDI. The shorter cycle time in the e-LFC $\mathrm{NB}_{\mathrm{N}}$ group is consistent with short-cycle periodic breathing being associated with NREM dominant sleep apnea and high loop gain (Gilmartin et al. 2005).

\section{A sleep fragmentation phenotype}

A distinct sleep fragmentation phenotype was evident at all severities of obstructive sleep apnea. This result is generally consistent with the variability of arousal phenomena in sleep apnea, contributing to amplification of disease, especially in NREM sleep (Eckert \& Younes 2014). While low arousal threshold is a measurable sleep apnea phenotype (Eckert et al. 2013), the return to sleep after arousal is probably just as important. Recovery from arousal is a continuous process of variable dynamics (Younes \& Hanly 2016; Younes et al. 2015), and if delayed, the epoch will be scored as wake or N1/S1. If this phenotype is a trait, sedatives may have a role in management, similar to reducing the apnea-hypopnea index in NREM sleep in those with low arousal threshold (Smales et al. 2015). As no physiological sleep apnea trait estimates were performed in the APPLES, concordance or discordance of a low arousal vs. sleep fragmentation phenotype could not be determined. High N1 fragmentation phenotype was associated with reduced compliance.

\section{A need for improved phenotyping}

From a diagnostic standpoint, there is minimal relationship of the AHI with subjective or objective sleepiness 
measurements (Gottlieb et al. 1999; Eiseman et al. 2012). From a treatment standpoint, predicting paradoxical PAP response (complex apnea) is not reliably predicted standard metrics, but is predicted by CPC metrics (Thomas et al. 2007a). Diagnostic phenotyping is important for appropriate clinical case detection, epidemiology, and clinical trial planning purposes. Treatment phenotypes should reasonably focus on response to therapy, positive pressure or otherwise. Persistence of phenotypes or conversion of one phenotype to another can have clinical implications. For example, a fragmentation phenotype which persists may benefit from sedatives, cognitive behavioral therapy or re-looking at therapeutic precision, while a fragmentation to consolidated phenotype shift implies therapeutic success. Therapies can target driving phenotypes to more desirable ones, e.g., acetazolamide for a high loop gain/NREM-dominant phenotype (Edwards et al. 2012).

\section{Phenotypes and clinical covariates}

The slightly lower subjective sleepiness score in NREM dominant sleep apnea is largely in keeping with lesser degrees of subjective sleepiness in patients with strong respiratory chemoreflex activation. Heart rate variability and muscle sympathetic nerve activity are inversely related to subjective sleepiness in heart failure (Taranto Montemurro et al. 2012; Taranto Montemurro et al. 2014). The tight link of the respiratory chemoreflex and sympathetic centers in the brainstem is one plausible explanation-that these individuals have heightened sympathetic drive for a given degree of sleep apnea. Increased hypertension risk in those with reduced high frequency coupling, a signal biomarker of stable breathing and sleep, could reflect the impact of longer periods of stable breathing and the associated vagal dominance, even in those with sleep apnea, on overall blood pressure control.

The relative lack of impact of the various phenotypes on any measure of cognition was a surprise. This result may reflect the characteristics of the APPLES population which resulted in a negative result in the primary study (CPAP vs. placebo CPAP). The mechanisms which impair cognition and mood in sleep apnea are not well understood, and likely reflect an interaction of the stressor and individual resilience factors. Our result may also reflect our current inability to identify the factors associated with a certain apnea-hypopnea index which determines an adverse impact on brain function. The APPLES follow-up data we aim to analyze may provide additional clues.

\section{Limitations of the analysis}

The primary limitation of the presented analysis is that the impact of phenotypes on outcomes cannot be determined. The criteria for various phenotype categories were necessarily arbitrary but are "clinically reasonable". Moreover, if these patterns are maintained over time despite positive pressure therapy is important to know, and will need further follow-up analysis of the APPLES data. Body position effects were not quantified. Respiratory Effort Related Arousal events were not scored in the APPLES, and could alter some of our conclusions. Careful scoring of periodic breathing without the filter of flow limitation may provide higher estimates of loop gain than standard scoring. A more detailed analysis of compliance metrics across the 6 months, including differentiating sham vs. real CPAP, will be required to establish an impact of phenotypes described here.

\section{Conclusions}

Distinct phenotypes of fragmentation, consolidation, NREM vs. REM dominance, and high loop gain can be identified in the conventional polysomnogram, by both standard scoring and estimates of cardiopulmonary coupling. Baseline clinical characteristics including cognition were not impacted by the phenotypes. The impact of these phenotypes on treatment clinical outcomes require analysis and research.

\section{Abbreviations}

AASM: American Academy of Sleep Medicine; APPLES: Apnea Positive Pressure Long-term Efficacy Study; BMI: Body mass index; CAl: Central apnea index; CPAP: Continuous positive airway pressure; CPC: Cardiopulmonary coupling; CSR: Cheyne-Stokes respiration; ECG: Electrocardiogram;

EEG: Electroencephalogram; HFC: High frequency coupling; LFC: Low frequency coupling; NREM: Non-rapid eye movement; PSG: Polysomnogram; RDI: Respiratory disturbance index; REM: rapid eye movement; SC: Sleep consolidation; SF: Sleep fragmentation; VLFC: Very low frequency coupling; WASO: Wake after sleep onset

\section{Acknowledgments}

Joseph Mietus, B.S, (deceased) developer of the original cardiopulmonary coupling software, and who performed some of the ECG analysis. APPLES

Acknowledgment List

APPLES was funded by contract 5UO1-HL-068060 from the National Heart, Lung and Blood Institute. The APPLES pilot studies were supported by grants from the American Academy of Sleep Medicine and the Sleep Medicine Education and Research Foundation to Stanford University and by the National Institute of Neurological Disorders and Stroke (N44-NS-002394) to SAM Technology.

In addition, APPLES investigators gratefully recognize the vital input and support of Dr. Sylvan Green who died before the results of this trial were analyzed, but was instrumental in its design and conduct. ADMINISTRATIVE CORE

Clete A. Kushida, M.D., Ph.D, Deborah A. Nichols, M.S., Eileen B. Leary, B.A., RPSGT, Pamela R. Hyde, M.A., Tyson H. Holmes, Ph.D., Daniel A. Bloch, Ph.D. William C. Dement, M.D., Ph.D.

DATA COORDINATING CENTER

Daniel A. Bloch, Ph.D., Tyson H. Holmes, Ph.D., Deborah A. Nichols, M.S., Rik Jadrnicek, Microflow, Ric Miller, Microflow, Usman Aijaz, M.S., Aamir Farooq, Ph.D., Darryl Thomander, Ph.D., Chia-Yu Cardell, RPSGT, Emily Kees, Michael E. Sorel, M.P.H., Oscar Carrillo, RPSGT, Tami Crabtree, M.S., Booil Jo, Ph.D., Ray Balise, Ph.D., Tracy Kuo, Ph.D.

CLINICAL COORDINATING CENTER

Clete A. Kushida, M.D., Ph.D., William C. Dement, M.D., Ph.D., Pamela R. Hyde, M.A., Rhonda M. Wong, B.A., Pete Silva, Max Hirshkowitz, Ph.D., Alan Gevins, 
D.Sc., Gary Kay, Ph.D., Linda K. McEvoy, Ph.D., Cynthia S. Chan, B.S., Sylvan Green, M.D.

\section{CLINICAL CENTERS}

\section{Stanford University}

Christian Guilleminault, M.D., Eileen B. Leary, B.A., RPSGT, David Claman, M.D., Stephen Brooks, M.D., Julianne Blythe, P.A.-C, RPSGT, Jennifer Blair, B.A., Pam Simi, Ronelle Broussard, B.A., Emily Greenberg, M.P.H., Bethany Franklin, M.S. Amirah Khouzam, M.A., Sanjana Behari Black, B.S., RPSGT, Viola Arias, RPSGT, Romelyn Delos Santos, B.S., Tara Tanaka, Ph.D.

University of Arizona

Stuart F. Quan, M.D., James L. Goodwin, Ph.D., Wei Shen, M.D., Phillip Eichling, M.D., Rohit Budhiraja, M.D., Charles Wynstra, M.B.A., Cathy Ward, Colleen Dunn, B.S., Terry Smith, B.S., Dane Holderman, Michael Robinson, B.S., Osmara Molina, B.S., Aaron Ostrovsky, Jesus Wences, Sean Priefert, Julia Rogers, B.S., Megan Ruiter, B.S., Leslie Crosby, B.S., R.N.

St. Mary Medical Center

Richard D. Simon, Jr., M.D., Kevin Hurlburt, RPSGT, Michael Bernstein, M.D., Timothy Davidson, M.D., Jeannine Orock-Takele, RPSGT, Shelly Rubin, M.A. Phillip Smith, RPSGT, Erica Roth, RPSGT, Julie Flaa, RPSGT, Jennifer Blair, B.A., Jennifer Schwartz, B.A., Anna Simon, B.A., Amber Randall, B.A.

\section{St. Luke's Hospital}

James K. Walsh, Ph.D., Paula K. Schweitzer, Ph.D., Anup Katyal, M.D., Rhody Eisenstein, M.D., Stephen Feren, M.D., Nancy Cline, Dena Robertson, R.N., Sheri Compton, R.N., Susan Greene, Kara Griffin, M.S., Janine Hall, Ph.D. Brigham and Women's Hospital

Daniel J. Gottlieb, M.D., M.P.H., David P. White, M.D., Denise Clarke, B.Sc RPSGT, Kevin Moore, B.A., Grace Brown, B.A., Paige Hardy, M.S., Kerry Eudy, Ph.D., Lawrence Epstein, M.D., Sanjay Patel, M.D. *Sleep HealthCenters for the use of their clinical facilities to conduct this research

\section{CONSULTANT TEAMS}

Methodology Team: Daniel A. Bloch, Ph.D., Sylvan Green, M.D., Tyson H. Holmes, Ph.D., Maurice M. Ohayon, M.D., D. Sc., David White, M.D., Terry Young, Ph.D.

Sleep-Disordered Breathing Protocol Team: Christian Guilleminault, M.D., Stuart Quan, M.D., David White, M.D.

EEG/Neurocognitive Function Team: Jed Black, M.D., Alan Gevins, D.Sc., Max Hirshkowitz, Ph.D., Gary Kay, Ph.D., Tracy Kuo, Ph.D.

Mood and Sleepiness Assessment Team: Ruth Benca, M.D., Ph.D., William C. Dement, M.D., Ph.D., Karl Doghramii, M.D., Tracy Kuo, Ph.D., James K. Walsh, Ph.D. Quality of Life Assessment Team: W. Ward Flemons, M.D., Robert M. Kaplan, Ph.D. ASA-NC Team: Dean Beebe, Ph.D., Robert Heaton, Ph.D., Joel Kramer, Psy.D., Ronald Lazar, Ph.D., David Loewenstein, Ph.D., Frederick Schmitt, Ph.D.

NATIONAL HEART, LUNG, AND BLOOD INSTITUTE (NHLBI)

Michael J. Twery, Ph.D., Gail G. Weinmann, M.D., Colin O. Wu, Ph.D.

\section{DATA AND SAFETY MONITORING BOARD (DSMB)}

Seven-year term: Richard J. Martin, M.D. (Chair), David F. Dinges, Ph.D. Charles F. Emery, Ph.D., Susan M. Harding M.D., John M. Lachin, Sc.D., Phyllis C. Zee, M.D., Ph.D.

Other term: Xihong Lin, Ph.D. (2 years), Thomas H. Murray, Ph.D. (1 year) INDUSTRY SUPPORT - Philips Respironics, Inc.

\section{Funding}

To Robert J. Thomas: RC1HL099749-01 (National Heart Lung Blood Institute, USA), Beth Israel Deaconess Medical Center Chief Academic Officer's Research Innovation Initiative

APPLES was funded by contract 5UO1-HL-068060 from the National Heart, Lung and Blood Institute. The

APPLES pilot studies were supported by grants from the American Academy of Sleep Medicine and the Sleep

Medicine Education and Research Foundation to Stanford University and by the National Institute of Neurological Disorders and Stroke (N44-NS-002394) to SAM Technology.

The funding bodies had no role in the design of the study and collection, analysis, and interpretation of data and in writing the manuscript.

\section{Availability of data and materials}

The datasets generated during and/or analysed during the current study are not publicly available, and were obtained from the APPLES investigators following a Data Agreement through the Beth Israel Deaconess Medical Center. Secondary distribution of the data is not permitted. Individuals who wish to obtain the data may initiate the process by contacting co-author Clete Kushida, M.D., the APPLES Principal Investigator.

\section{Authors' contributions}

RJT, MTB and CY conceptualized the analysis. RJT performed the analysis and wrote the manuscript draft. CK provided access to and interpretation assistance for the APPLES data. All authors contributed to revision of the manuscript draft. All authors read and approved the final manuscript.

\section{Competing interests}

RJT reports the following: 1) Patent and license for a method to use the ECG to phenotype sleep and sleep apnea; 2) Patent to treat central and complex sleep apnea using low concentration carbon dioxide; 3) Patent submitted and licensed software in an auto-CPAP, joint work with DeVilbiss-DRIVE' 4) General sleep medicine consulting with GLG Councils.

MTB reports the following: Funding from the Center for Integration of Medicine and Innovative Technology, the Milton Family Foundation, and currently receives funding from the Department of Neurology, the MGH-MIT Grand Challenge, and the American Sleep Medicine Foundation. He has a patent pending on a home sleep monitoring device. He has consulting and research agreements with $\mathrm{MC10}$, Insomnisolv, and McKesson. He has provided expert testimony in sleep medicine.

The other authors have no declared conflicts of interest.

\section{Consent for publication}

Not Applicable. The APPLES data is de-identified, and individuals are not recognizable through the sleep hypnogram.

\section{Ethics approval and consent to participate}

Institutional Review Board Approval from the Beth Israel Deaconess Medical Center and required Data Transfer and Use Agreements were obtained prior to the analysis.

\section{Publisher's Note}

Springer Nature remains neutral with regard to jurisdictional claims in published maps and institutional affiliations.

\section{Author details}

${ }^{1}$ Division of Pulmonary, Critical Care, and Sleep Medicine, Beth Israel Deaconess Medical Center, Boston, MA, USA. ${ }^{2}$ Institute of Human Genomic Study, Department of Respiratory Internal Medicine, Korea University Ansan Hospital, Ansan, South Korea. ${ }^{3}$ Division of Sleep Medicine, Department of Neurology, Massachusetts General Hospital, Boston, MA, USA. ${ }^{4}$ Psychiatry and Behavioral Sciences, Stanford Center for Sleep Sciences and Medicine, Stanford University Medical Center, Redwood City, CA, USA. ${ }^{5}$ Department of Neurology, Seoul National University Bundang Hospital, Seongnam, South Korea.

Received: 9 August 2016 Accepted: 9 March 2017

Published online: 10 May 2017

\section{References}

Badr MS. Effect of ventilatory drive on upper airway patency in humans during NREM sleep. Respir Physiol. 1996;103:1-10.

Badr MS, Toiber F, Skatrud JB, Dempsey J. Pharyngeal narrowing/occlusion during central sleep apnea. J Appl Physiol. 1995;78:1806-15.

Berry RB BR, Gamaldo CE, Harding SM, Lloyd RM, Marcus CL and Vaughn BV for the American, Academy of Sleep Medicine. The AASM Manual for the Scoring of Sleep and Associated Events: Rules, Terminology and Technical Specifications VwaoD, Illinois: American Academy of Sleep Medicine. 2017 Version 2.4 .

Dempsey JA, Xie A, Patz DS, Wang D. Physiology in medicine: obstructive sleep apnea pathogenesis and treatment-considerations beyond airway anatomy. J Appl Physiol. 2014;116:3-12.

Dowdell WT, Javaheri S, McGinnis W. Cheyne-Stokes respiration presenting as sleep apnea syndrome. Clinical and polysomnographic features. Am Rev Respir Dis. 1990;141:871-9.

Eckert DJ, Younes MK. Arousal from sleep: implications for obstructive sleep apnea pathogenesis and treatment. J Appl Physiol (1985). 2014;116:302-13.

Eckert DJ, Jordan AS, Merchia P. Malhotra A. Central sleep apnea: Pathophysiology and treatment. Chest. 2007;131:595-607. 
Eckert DJ, White DP, Jordan AS, Malhotra A, Wellman A. Defining phenotypic causes of obstructive sleep apnea. Identification of novel therapeutic targets. Am J Respir Crit Care Med. 2013;188:996-1004.

Edwards BA, Sands SA, Eckert DJ, et al. Acetazolamide improves loop gain but not the other physiological traits causing obstructive sleep apnoea. J Physiol. 2012;590:1199-211.

Eiseman NA, Westover MB, Mietus JE, Thomas RJ, Bianchi MT. Classification algorithms for predicting sleepiness and sleep apnea severity. J Sleep Res. 2012;21:101-12.

Gilmartin GS, Daly RW, Thomas RJ. Recognition and management of complex sleep-disordered breathing. Curr Opin Pulm Med. 2005;11:485-93.

Gottlieb DJ, Whitney CW, Bonekat WH, et al. Relation of sleepiness to respiratory disturbance index: the Sleep Heart Health Study. Am J Respir Crit Care Med. 1999;159:502-7

Guilleminault C, Hill MH, Simmons FB, Powell N, Riley R, Stoohs R. Passive constriction of the upper airway during central apneas: fiberoptic and EMG investigations. Respir Physiol. 1997;108:11-22.

Hanly PJ, Millar TW, Steljes DG, Baert R, Frais MA, Kryger MH. Respiration and abnormal sleep in patients with congestive heart failure. Chest. 1989:96:480-8.

Huang Y, Mai W, Cai $X$, et al. The effect of zolpidem on sleep quality, stress status, and nondipping hypertension. Sleep Med. 2012;13:263-8.

Iber C, American Academy of Sleep Medicine. The AASM manual for the scoring of sleep and associated events: rules, terminology, and technical specifications. Westchester: American Academy of Sleep Medicine; 2007.

Javaheri S, Dempsey JA. Central sleep apnea. Compr Physiol. 2013;3:141-63.

Jordan AS, White DP, Lo YL, et al. Airway dilator muscle activity and lung volume during stable breathing in obstructive sleep apnea. Sleep. 2009;32:361-8.

Kara T, Narkiewicz K, Somers VK. Chemoreflexes-physiology and clinical implications. Acta Physiol Scand. 2003;177:377-84.

Kushida CA, Nichols DA, Quan SF, et al. The Apnea Positive Pressure Long-term Efficacy Study (APPLES): rationale, design, methods, and procedures. J Clin Sleep Med. 2006:2:288-300.

Maestri R, La Rovere MT, Robbi E, Pinna GD. Fluctuations of the fractal dimension of the electroencephalogram during periodic breathing in heart failure patients. J Comput Neurosci. 2010;28:557-65.

Owens RL, Edwards BA, Eckert DJ, et al. An integrative model of physiological traits can be used to predict obstructive sleep apnea and response to non positive airway pressure therapy. Sleep. 2015;38:961-70.

Parrino L, Boselli M, Spaggiari MC, Smerieri A, Terzano MG. Multidrug comparison (lorazepam, triazolam, zolpidem, and zopiclone) in situational insomnia: polysomnographic analysis by means of the cyclic alternating pattern. Clin Neuropharmacol. 1997;20:253-63.

Parrino L, Grassi A, Milioli G. Cyclic alternating pattern in polysomnography: what is it and what does it mean? Curr Opin Pulm Med. 2014;20:533-41.

Quadri S, Drake C, Hudgel DW. Improvement of idiopathic central sleep apnea with zolpidem. J Clin Sleep Med. 2009;5:122-9.

Quan SF, Chan CS, Dement WC, et al. The association between obstructive sleep apnea and neurocognitive performance-the Apnea Positive Pressure Longterm Efficacy Study (APPLES). Sleep. 2011;34:303-14B.

Rao H, Thomas RJ. Complex sleep apnea. Curr Treat Options Neurol. 2013;15:677-91.

Sankri-Tarbichi AG, Rowley JA, Badr MS. Expiratory pharyngeal narrowing during central hypocapnic hypopnea. Am J Respir Crit Care Med. 2009;179:313-9.

Smales ET, Edwards BA, Deyoung PN, et al. Trazodone effects on obstructive sleep apnea and non-rem arousal threshold. Ann Am Thorac Soc. 2015;12:758-64.

Stanchina M, Robinson K, Corrao W, Donat W, Sands S, Malhotra A. Clinical use of loop gain measures to determine continuous positive airway pressure efficacy in patients with complex sleep apnea. A pilot study. Ann Am Thorac Soc. 2015;12:1351-7.

Taranto Montemurro L, Floras JS, Millar PJ, et al. Inverse relationship of subjective daytime sleepiness to sympathetic activity in patients with heart failure and obstructive sleep apnea. Chest. 2012;142:1222-8.

Taranto Montemurro L, Floras JS, Picton P, et al. Relationship of heart rate variability to sleepiness in patients with obstructive sleep apnea with and without heart failure. J Clin Sleep Med. 2014;10:271-6.

Terrill PI, Edwards BA, Nemati S, et al. Quantifying the ventilatory control contribution to sleep apnoea using polysomnography. Eur Respir J. 2015;45:408-18.

Terzano MG, Mancia D, Salati MR, Costani G, Decembrino A, Parrino L. The cyclic alternating pattern as a physiologic component of normal NREM sleep. Sleep. 1985:8:137-45.

Terzano MG, Parrino L, Boselli M, Dell'Orso S, Moroni M, Spaggiari MC. Changes of cyclic alternating pattern (CAP) parameters in situational insomnia under brotizolam and triazolam. Psychopharmacology (Berl). 1995;120:237-43.
Thomas RJ. Cyclic alternating pattern and positive airway pressure titration. Sleep Med. 2002;3:315-22.

Thomas RJ. Alternative approaches to treatment of Central Sleep Apnea. Sleep Med Clin. 2014:9:87-104.

Thomas RJ, Mietus JE, Peng CK, Goldberger AL. An electrocardiogram-based technique to assess cardiopulmonary coupling during sleep. Sleep. 2005;28:1151-61.

Thomas RJ, Mietus JE, Peng CK, et al. Differentiating obstructive from central and complex sleep apnea using an automated electrocardiogram-based method. Sleep. 2007a;30:1756-69.

Thomas RJ, Tamisier R, Boucher J, et al. Nocturnal hypoxia exposure with simulated altitude for 14 days does not significantly alter working memory or vigilance in humans. Sleep. 2007b;30:1195-203.

Thomas RJ, Weiss MD, Mietus JE, Peng CK, Goldberger AL, Gottlieb DJ. Prevalent hypertension and stroke in the Sleep Heart Health Study: association with an ECG-derived spectrographic marker of cardiopulmonary coupling. Sleep. 2009;32:897-904

Thomas RJ, Mietus JE, Peng CK, et al. Relationship between delta power and the electrocardiogram-derived cardiopulmonary spectrogram: possible implications for assessing the effectiveness of sleep. Sleep Med. 2014;15:125-31.

Walker JM, Farney RJ, Rhondeau SM, et al. Chronic opioid use is a risk factor for the development of central sleep apnea and ataxic breathing. J Clin Sleep Med. 2007:3:455-61.

Weiss MD, Tamisier R, Boucher J, et al. A pilot study of sleep, cognition, and respiration under 4 weeks of intermittent nocturnal hypoxia in adult humans. Sleep Med. 2009;10:739-45.

Wellman A, Malhotra A, Jordan AS, Stevenson KE, Gautam S, White DP. Effect of oxygen in obstructive sleep apnea: role of loop gain. Respir Physiol Neurobiol. 2008;162:144-51.

Wellman A, Edwards BA, Sands SA, et al. A simplified method for determining phenotypic traits in patients with obstructive sleep apnea. J Appl Physiol (1985). 2013;114:911-22.

Xie A, Bedekar A, Skatrud JB, Teodorescu M, Gong Y, Dempsey JA. The heterogeneity of obstructive sleep apnea (predominant obstructive vs pure obstructive apnea). Sleep. 2011;34:745-50.

Younes M. Hanly PJ. Immediate postarousal sleep dynamics: an important determinant of sleep stability in obstructive sleep apnea. J Appl Physiol (1985). 2016:120:801-8.

Younes M, Ostrowski M, Soiferman M, et al. Odds ratio product of sleep EEG as a continuous measure of sleep state. Sleep. 2015:38:641-54.

\section{Submit your next manuscript to BioMed Central and we will help you at every step:}

- We accept pre-submission inquiries

- Our selector tool helps you to find the most relevant journal

- We provide round the clock customer support

- Convenient online submission

- Thorough peer review

- Inclusion in PubMed and all major indexing services

- Maximum visibility for your research

Submit your manuscript at www.biomedcentral.com/submit
) Biomed Central 